\section{Einsatzbeispiele und Scale-up von Wirbelschichtreaktoren mit Kugeln aus porösem Sinterglas in der anaeroben Abwasserreinigung*}

\section{Peter Keim, Markus Luerweg, Bernd Striegel, Alexander Aivasidis und Christian Wandrey**}

\section{Problemstellung}

Ein Beitrag zur Reaktions- und Verfahrenstechnik anaerober Wirbelschichtreaktoren mit makroporösem Trägermaterial sollte geleistet werden, wozu die Konstruktion und der Bau von Labor-Wirbelschichtreaktoren und deren hydrodynamische Optimierung gehören. Mit den im Labormaßstab gewonnenen Erfahrungen und Auslegungskriterien sollte dann anschließend ein Scale-up durchgeführt werden. Bei der Entwicklung der Wirbelschichttechnik unter Einsatz dieses Trägermaterials kommt der Verteilerkonstruktion am Reaktorboden eine bedeutende Funktion zu, da die Güte der Flüssigkeitsverteilung über den gesamten Reaktorquerschnitt sowohl über die Homogenität des Partikelbetts als auch über den dazu notwendigen Energieeintrag entscheidet. Bei der hier vorliegenden quasi Dreiphasen-Wirbelschicht tritt noch eine zusätzliche Schwierigkeit auf, da die Fluidisierung des Trägermaterials mittels der flüssigen Phase und nicht wie sonst üblich durch die Gasphase erfolgen muß.

\section{Lösungsansatz}

Dazu wurden zunächst einmal in Labor-Wirbelschichtreaktoren aus Glas mit einem Gesamtvolumen von 451 verschiedene Verteilersysteme anhand vorher aufgestellter Kriterien auf ihre Eignung hin überprüft [1]. In Abb. 1 sind die unterschiedlichen untersuchten Verteilersysteme zusammengestellt. Die prozentuale Wirbelbett-Ausdehnung eines bestimmten Trägervolumens bei stets gleichbleibender Strömungsgeschwindigkeit im Wirbelschichtreaktor diente als $\mathrm{MaB}$ für die Qualität des untersuchten Verteilersystems. Befriedigende Ergebnisse konnten nur mit dem selbstkonstruierten Verteilersystem, dem
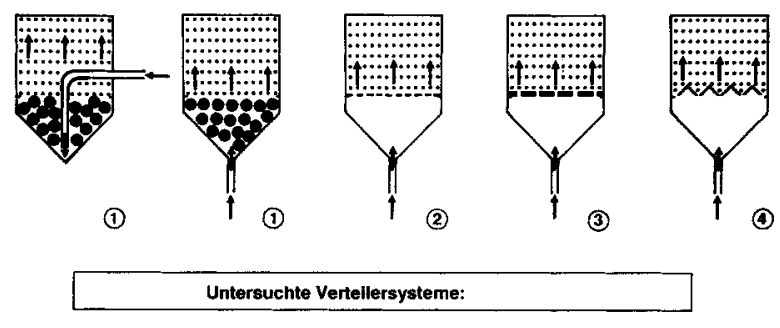

\footnotetext{
(1) Schültungen aus Glaskugeln unterschiedlicher Durchmesser.

(2) Gitternetze mit verschiedenen Maschenweilen.

(3) Lochplatten mitt unterschiedlicher Anzahl, Größe und Auftellung der Löcher.

(4) Parallelechlitz-Plattenvertellerboden.
}

Abb. 1. Darstellung der untersuchten Verteilersysteme für den Einsatz im Labor-Wirbelschichtreaktor.

* Vortrag von P. Keim auf dem Jahrestreffen der VerfahrensIngenieure, 27. bis 29. Sept. 1989 in Berlin.

** Dipl.-Chem. P. Keim, Dipl.-Ing. M. Luenweg, Dipl.-Ing. B. Striegel, Dr. A. Aivasidis und Prof. Dr. C. Wandrey, Kernforschungsanlage Jülich, Institut für Biotechnologie 2, 5170 Jülich.
Parallelschlitz-Plattenverteilerboden, erzielt werden, so daß dieser auch für die nachfolgenden Experimente mit Industrieabwässern eingesetzt wurde (Abb. 2).

\section{Ergebnisse}

Mit Hilfe einer rechnergekoppelten Regelungstechnik konnten bei allen Labor-Wirbelschichtreaktoren ein zeitoptimaler Anfahrvorgang und eine hohe Prozeßsicherheit erreicht werden. Dazu wurden die wichtigsten Prozeßdaten, wie Substratdurchfluß, Umlaufmenge, pHWert, Biogasmenge und -zusammensetzung, mittels Dateninterface und PC on-line erfaßt. Als Trägermaterial wurden 101 Kugeln aus offenporigem Sinterglas, welches von den Schott Glaswerken, Mainz, hergestellt wird, in den Labor-Wirbelschichtreaktoren eingesetzt. Bislang wurden folgende praktische Industrieabwässer in den LaborWirbelschichtreaktoren untersucht:

Zellstoffindustrie: Brüdenkondensate;

Brauereien: Mischabwasser aus der Flaschen- und Faßreinigung sowie Preßsäfte der Naßtreber;

Altpapierindustrie: Kreislaufwasser;

Hefeindustrie: Hefeabwürze.

Zellstoffindustrie: Brüdenkondensate aus der Zellstoffindustrie sind aufgrund ihres destillierten Charakters weitgehend feststofffreie, aber organisch hoch belastete Abwässer und werden gerne aufgrund ihrer einfachen Zusammensetzung für die ersten Untersuchungen eingesetzt. Folgende Daten konnten erzielt werden: $\mathrm{CSB}_{\text {ein }}: 50,2 \mathrm{~kg} / \mathrm{m}^{3}$;

CSB-Umsatz: $79,0 \%$;

Verweilzeit: 3,8 h (bez. auf Totalvolumen); Raumbelastung: bis zu $314 \mathrm{~kg} \mathrm{CSB} /\left(\mathrm{m}^{3} \mathrm{~d}\right)$; Raum-Zeit-Ausbeute: bis zu $248 \mathrm{~kg} \mathrm{CSB} /\left(\mathrm{m}^{3} \mathrm{~d}\right)$; Biogasproduktion: bis zu $160 \mathrm{~m}^{3} /\left(\mathrm{m}^{3} \mathrm{~d}\right)$.

Vergleichbar hohe CSB-Eliminationsraten sind bislang aus der Literatur nicht bekannt geworden.

Brauereien: Mischabwässer aus Brauereien bieten sich aufgrund ihres hohen Feststoffgehalts von bis $z u g g / l$ besonders gut für den Einsatz in Wirbelschichtreaktoren an, da andere Reaktorsysteme schnell versagen. Folgende Daten konnten erzielt werden: $\mathrm{CSB}_{\text {ein }}: 8,8$ bis $11,0 \mathrm{~kg} / \mathrm{m}^{3}$;

CSB-Umsatz: 70,0\%;

Verweilzeit: $3,4 \mathrm{~h}$;
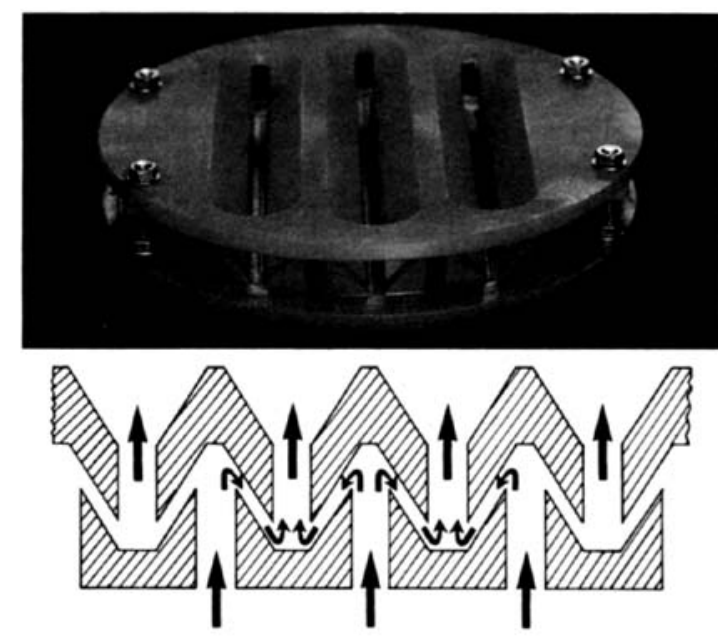

Abb. 2. Darstellung des Parallelschlitz-Plattenverteilerbodens, wie er im Labormaßstab eingesetzt wird, und eine schematische Darstellung der Funktionsweise. 


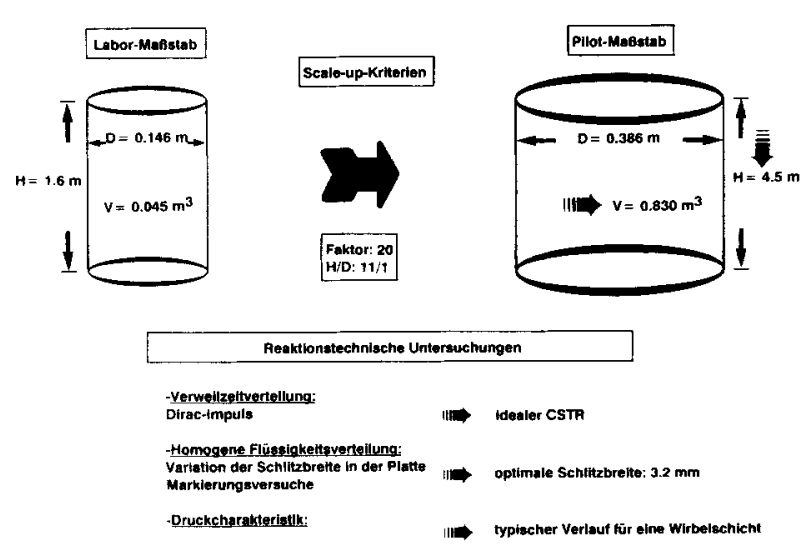

Abb. 3. Schematische Darstellung der Maßstabsvergrößerung und der reaktionstechnischen Untersuchungen im Pilot-Wirbelschichtreaktor.

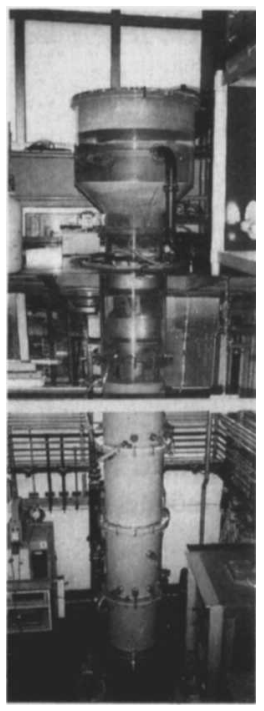

Abb. 4. Foto des Pilot-Wirbelschichtreaktors im Biotechnikum des IBT.

Raumbelastung: bis zu $80 \mathrm{~kg} \mathrm{CSB} /\left(\mathrm{m}^{3} \mathrm{~d}\right)$;

Raum-Zeit-Ausbeute: bis zu $60 \mathrm{~kg} \mathrm{CSB} /\left(\mathrm{m}^{3} \mathrm{~d}\right)$;

Biogasproduktion: bis zu $39 \mathrm{~m}^{3} /\left(\mathrm{m}^{3} \mathrm{~d}\right)$.

Diese Leistung liegt um den Faktor 4 höher als die in einem Festbett-

Umlaufreaktor mit dem gleichen Abwasser erzielten Werte.

Altpapierindustrie: Kreislaufwasser aus dem Altpapier-Recycling enthält neben Feststoffen hohe Konzentrationen an Calcium, welches in Verbindung mit dem $\mathrm{CO}_{2}$ aus dem Biogas als Calciumcarbonat ausfallen kann und so den Reaktor, speziell das Verteilersystem oder die Oberfläche des Trägermaterials, blockieren kann. Durch den Einsatz eines Wirbelschichtreaktors und dessen Betrieb im sauren $\mathrm{pH}-$ Bereich des optimalen mikrobiellen Wachstums von 6,4 konnte ein Reaktorbetrieb von nun 17 Monaten ohne Störungen aufrecht erhalten werden. Dabei wurden folgende Daten erzielt:

$\mathrm{CSB}_{\text {ein }}: 9,5 \mathrm{~kg} / \mathrm{m}^{3}$;

CSB-Umsatz: $86,5 \%$;

Verweilzeit: $3,7 \mathrm{~h}$;

Raumbelastung: bis zu $62 \mathrm{~kg} \mathrm{CSB} /\left(\mathrm{m}^{3} \mathrm{~d}\right)$;

Raum-Zeit-Ausbeute: bis zu $54 \mathrm{~kg} \mathrm{CSB} /\left(\mathrm{m}^{3} \mathrm{~d}\right)$;

Biogasproduktion: bis zu $35 \mathrm{~m}^{3} /\left(\mathrm{m}^{3} \mathrm{~d}\right)$.

Die erzielten CSB-Eliminationsraten liegen um den Faktor 3 bis 5 höher als die aus der Literatur bekannten Werte.
Hefeindustrie: Mischabwässer aus der Hefeindustrie - hier ein Gemisch aus Hefeabwürze und Hefewaschwasser - können bis zu $4 \mathrm{~g}$ Sulfat/l enthalten. Da aber das Biogas schnell aus dem Reaktorsystem entweicht, traten bisher keinerlei hemmende Einflüsse auf. Folgende Daten konnten erzielt werden:

$\mathrm{CSB}_{\text {ein }}: 17,5 \mathrm{~kg} / \mathrm{m}^{3}$;

CSB-Umsatz: $62,0 \%$

Verweilzeit: $5,6 \mathrm{~h}$;

Raumbelastung: bis zu $75 \mathrm{~kg} \mathrm{CSB} /\left(\mathrm{m}^{3} \mathrm{~d}\right)$;

Raum-Zeit-Ausbeute: bis zu $46 \mathrm{~kg} \mathrm{CSB} /\left(\mathrm{m}^{3} \mathrm{~d}\right)$;

Biogasproduktion: bis zu $36 \mathrm{~m}^{3} /\left(\mathrm{m}^{3} \mathrm{~d}\right)$.

Ein höherer CSB-Umsatz konnte nicht erzielt werden, da aufgrund der Verwendung von Melasse als Substrat bei der Hefeproduktion ca. $30 \%$ der Abwasserbestandteile biologisch sehr schwer abbaubar sind.

\section{Scale-up}

Aufgrund der Erfahrungen mit den überzeugenden Ergebnissen beim Betrieb der Labor-Wirbelschichtreaktoren erfolgte eine Maßstabsvergrößerung um den Faktor 20 auf der Basis geometrischer Ähnlichkeit und unter Einhaltung eines $H / D$-Verhältnisses von 11:1 gegenüber den Laboranlagen. Der Pilot-Wirbelschichtreaktor wurde zunächst reaktionstechnisch charakterisiert (Abb. 3 und 4) und anschließend biologisch mit einem Brüdenkondensat der Zellstoffindustrie in Betrieb genommen.

Nach einer Versuchszeit von ca. 7 Monaten und Einstellung stationärer Verhältnisse konnte lediglich eine maximale erreichbare RaumZeit-Ausbeute von $90 \mathrm{~kg} \mathrm{CSB} /\left(\mathrm{m}^{3} \mathrm{~d}\right)$ erreicht werden, obwohl im Labormaßstab problemlos der doppelte Wert erzielt wurde. Nach Ausschließung möglicher limitierender Einflüsse durch die Nährstoffund Spurenelement-Konzentration sowie die im Reaktor vorhandene Biomasse konnte aus der Begrenzung der Raum-Zeit-Ausbeute gefolgert werden, da $B$ eine Maßstabsvergrößerung nur auf der Basis der geometrischen Ähnlichkeit nicht möglich ist. Weitere Scale-upKriterien müssen darüber hinaus berücksichtigt werden. Solch ein relevantes Kriterium ist die flächenbezogene Biogasbildung. Diese bereits früher bei Festbett-Umlaufreaktoren als höhenbegrenzender Faktor erkannte Größe kann aus einer Betrachtung zum Gas-Hold-up extrapoliert werden. Demnach beträgt die maximale flächenbezogene Biogasbelastung in den hier betrachteten Wirbelschichtreaktoren ca. $20 \mathrm{~m}^{3} /\left(\mathrm{m}^{2} \mathrm{~h}\right)$. Unter diesen Bedingungen sind die Trägerpartikel im oberen Teil der Wirbelschicht weitgehend von Biogas umgeben, und die Substratversorgung der Mikroorganismen wird zum limitierenden Faktor.

Eingegangen am 2. November 1989

\section{Literatur}

[1] Keim, P.; Luenweg, M.; Aivasidis, A.; Wandrey, C.: Korrespondenz Abwasser (1989) Nr. 6, S. 675/687.

Schlüsselworte: Anaerobe Abwasserreinigung, Wirbelschichtreaktor, offenporiges Sinterglas, Verteilersystem, Zellstoffindustrie, Brauerei, Altpapierindustrie, Hefeindustrie, Scale-up.

Das vollständige Manuskript dieser Arbeit Umfaßt 44 Seiten mit 25 Abbildungen, 1 Tabelle und 12 Literaturzitaten. Es ist als Fotokopie oder Mikrofiche MS 1855/90 erhältlich. 